Notre groupe de recherche, récemment créé au Laboratoire de Physique des Solides d'Orsay, développe de nouveaux modes de diffusion et de vulgarisation de la physique.

Des collaborations étroites entre physiciens et créateurs (graphistes, illustrateurs, designers...) permettent ainsi d'explorer de nouveaux formats pour montrer autrement la physique quantique et le monde de la recherche au grand public : animations pédagogiques, dispositifs ludiques, illustrations, vidéos, ou objets de design.

L'objectif est double : créer des outils utiles à tous et de qualité professionnelle, et aussi convaincre plus de chercheurs de participer à des actions de vulgarisation.

\title{
Donner à voir la physique autrement
}

Julien Bobroff (julien.bobroff@u-psud.fr)

Laboratoire de Physique des Solides, Université Paris Sud et CNRS, 91405 Orsay

\section{Un cirque supraconducteur à la fac}

Fac d'Orsay, l'heure du déjeuner, l'amphi est bondé. Plus de quatre cents personnes, étudiants, chercheurs ou simples curieux, sont réunies pour assister au premier Science Break, un nouveau format de conférence de vulgarisation. C'est notre tour : avec mon collègue Frédéric Bouquet, nous avons dix minutes chrono pour présenter la supraconductivité à travers une expérience originale en direct. Nous déballons notre Physics Circus, petit cirque conçu avec un designer pour mettre en scène les différentes formes de lévitations supraconductrices grâce à de petits oiseaux en bois (fig. 1). Dix minutes plus tard, pari gagné, nous avons pu expérimenter, expliquer, et aussi émerveiller avec la physique quantique et ses manifestations macroscopiques. La vidéo suivra, pour être partagée avec le plus grand nombre sur internet.

Des nouvelles formes de vulgarisation, à l'exemple de ce Science Break, nous en avons testé pour la première fois en 2011, quand, avec l'Institut de Physique du CNRS, nous avons organisé l'Année de la supraconductivité. Cette année a été riche en enseignements. Elle nous a d'abord confirmé la curiosité et l'appétence du public, de tous les publics, pour la physique fondamentale et ses chercheurs. Nous avons aussi découvert, à l'occasion de la centaine d'évènements organisés partout en France, que la vulgarisation a tout à gagner à diversifier la nature de ses modes d'action vers le grand public. Plus que des expositions et conférences classiques, le succès est venu des dispositifs plus originaux, comme la maquette de Tour Eiffel en lévitation (voir les figures p. 25) ou le MagSurf, qui permet aux visiteurs de surfer eux-mêmes sur plusieurs mètres. Nous, physiciens, sommes arrivés à un moment clé où nous devons diversifier nos façons de vulgariser et d'engager le dialogue avec le grand public. Il faut explorer de nouveaux formats adaptés aux nouveaux publics, habitués à plus d'interactivité et à des formats courts répandus sur internet. Cependant, innover et produire des actions de qualité en mesure d'attirer une audience de plus en plus sollicitée demande du travail et du temps. Pour avoir un réel impact, ce type d'action ne peut plus être fait uniquement "à la marge " : cela demande un réel engagement de la part des personnes et des institutions. Sans quoi ce seront d'autres, communicants, professionnels des médias ou vidéastes amateurs, qui le feront à notre place, au risque de perdre sur le fond, sur la rigueur, mais aussi sur la vision qu'aura le grand public de ce qu'est la recherche.

Voilà pourquoi nous avons, Frédéric Bouquet et moi-même, créé en 2013 un groupe de recherche intitulé «La Physique Autrement ". Un groupe traditionnel par son fonctionnement : des physiciens, au sein d'un laboratoire de physique (le Laboratoire de Physique des Solides d'Orsay), avec des publications, des communications, des collaborations internationales, et des financements de recherche type ANR ou Chaire universitaire. Mais nouveau par son sujet de recherche : la vulgarisation en physique, abordée d'un point de vue interdisciplinaire. Il ne s'agit ni de remplacer les services de communication, ni de se substituer aux champs de recherche développés par les sciences humaines sur les rapports aux publics. Nous adoptons plutôt un point de vue de physiciens ancrés dans leur communauté et leur domaine, tout en développant des collaborations interdisciplinaires. Notre objectif est d'inventer de nouveaux modes de vulgarisation de la physique et des découvertes récentes, en particulier dans notre spécialité, la matière condensée. 


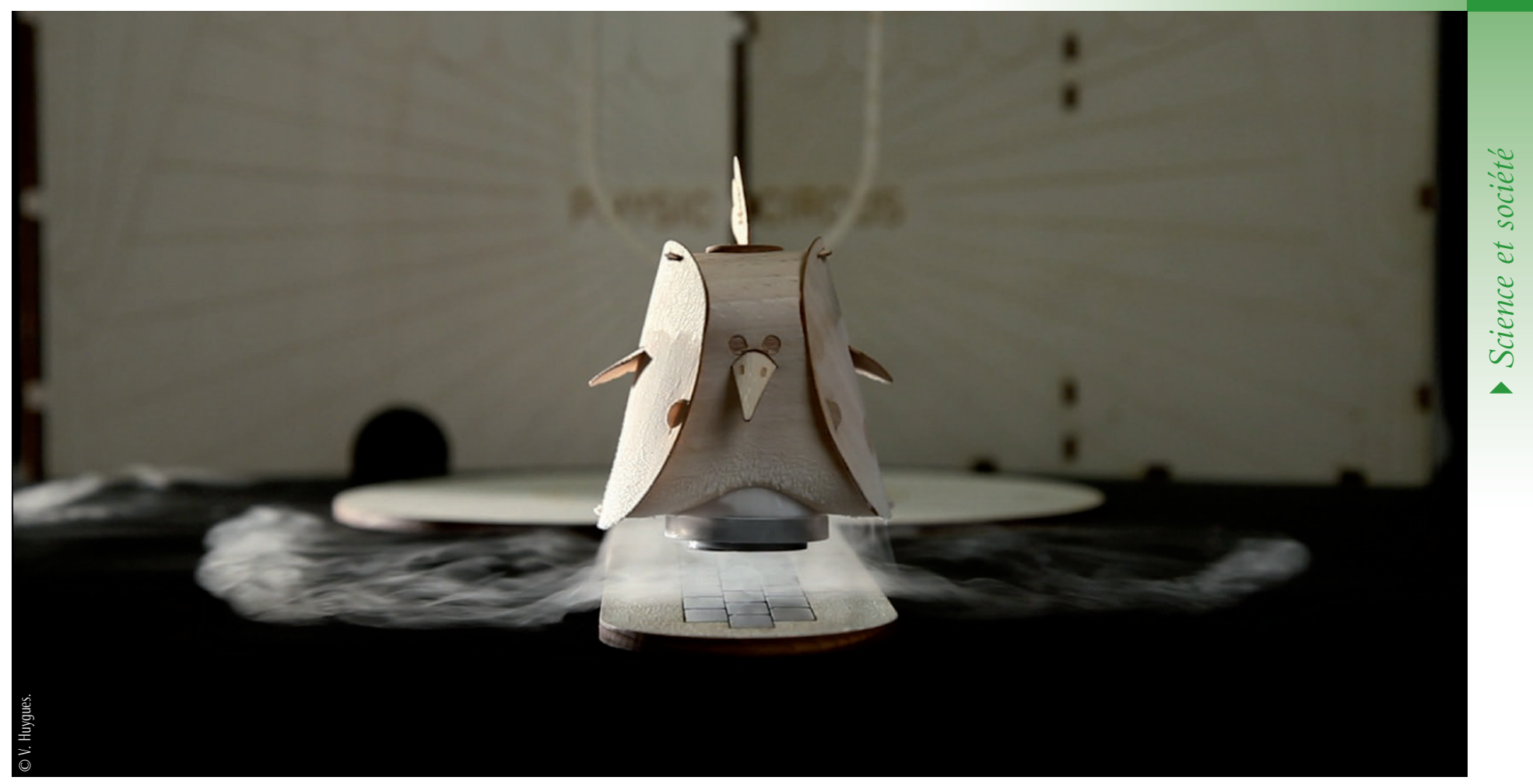

1. Le Physics Circus, conçu avec le designer A. Echasseriau, propose une version ludique de la supraconductivité. Ici, un oiseau en bois, dont la base est un supraconducteur qui lévite au-dessus d’un rail aimanté.

\section{Du design, du dessin, des animations}

La production de dispositifs originaux par leurs formats ou leurs contenus est sans doute la part la plus visible de notre démarche. Depuis deux ans, nous développons, par exemple avec des graphistes, une série d'animations sur la physique quantique, regroupées sur un site web www.ToutEstQuantique.fr (voir encadré). Ces petites vidéos pourraient sembler assez classiques. Mais leur création n'a été rendue possible que grâce à une collaboration étroite associant des graphistes et des collègues chercheurs spécialistes des sujets abordés. Un tel travail, avec implication forte des physiciens, n'aurait pu être mené de la même façon par un service de communication ou une agence multimédia.

Le site internet et les animations sont devenus des références pour les enseignants ou le grand public, à l'image du site www.supraconductivite.fr que nous avions réalisé sur la supraconductivité. Présentes également sur Wikipédia pour illustrer les articles correspondants, ces animations librement téléchargeables sont largement utilisées par des professeurs de lycées et de classes préparatoires pour illustrer leurs cours, et même par des chercheurs en France ou à l'étranger (tout est également disponible en anglais).

\section{Des animations quantiques}

Pour aider le grand public à mieux visualiser les propriétés propres à la physique quantique, nous avons développé avec une agence de graphisme des animations d'un format court (2 minutes). Sept animations décrivent les bases de la physique quantique : dualité, quantification, atomes, effet tunnel (fig. E1), spin, métal, laser. Nous venons de finir une deuxième série décrivant dans le même univers graphique des sujets ou techniques plus modernes : condensation de Bose-Einstein, graphène, décohérence quantique, technique pompe-sonde, photoémission et cristallographie en matière condensée ou liquide. Une troisième série portera bientôt sur les différentes formes de microscopies.

Chaque animation, conçue avec des scientifiques spécialistes du domaine, utilise un graphisme épuré et des textures tentant de représenter au mieux la nature quantique de la matière. Des légendes aident à la compréhension ; elles peuvent être enlevées pour permettre une utilisation en conférence. Une bande son souligne certains effets. La cohérence graphique entre animations permet de créer des liens entre les sujets, par exemple entre la nature ondulatoire de l'électron, la quantification et la classification périodique.

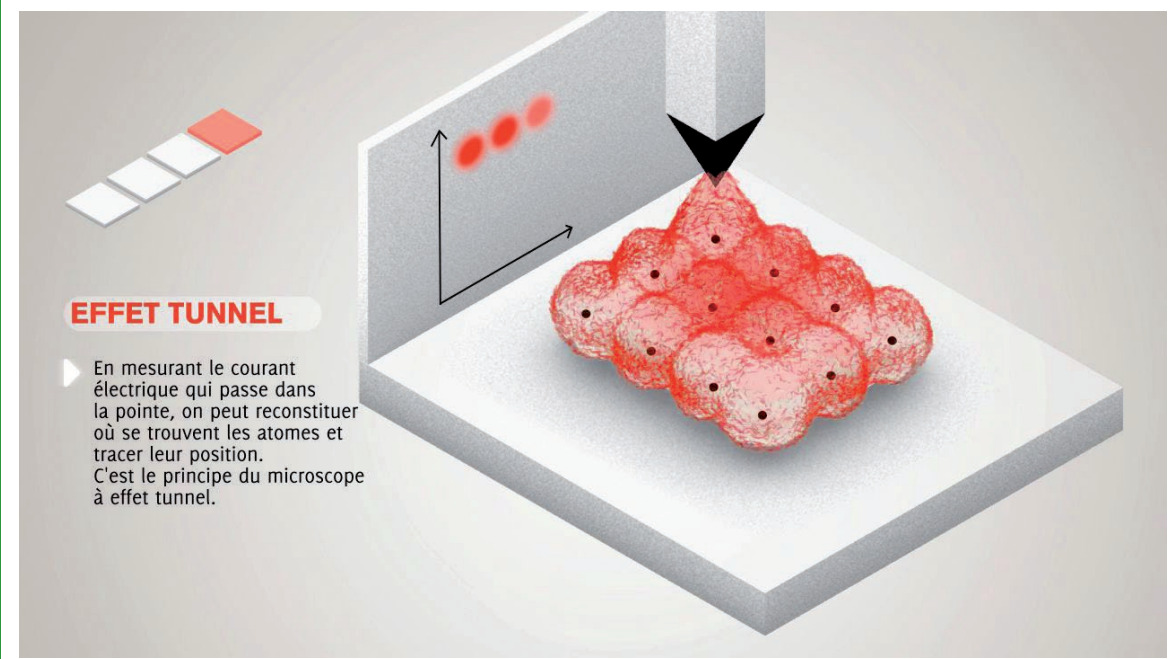

E1. Une des animations du site www.ToutEstQuantique.fr 
>>

Autre exemple : le projet « Physiciens des solides ", mené par une illustratrice qui a interrogé trois chercheurs de notre laboratoire sur leur vie quotidienne, leurs motivations et leurs recherches. Elle en a tiré une série d'illustrations, regroupées sous forme de livrets et de cartes postales (fig. 2). Chaque livret est accompagné d'un encart expliquant la physique en jeu : films de savon, magnétisme quantique, cônes de Dirac dans le graphène. Ces livrets, distribués auprès des scolaires, donnent à voir non seulement des sujets de physique moderne, mais aussi une autre image des chercheurs, plus sensible, plus humaine.

Pour décrire une autre facette du monde de la recherche, nous avons conçu avec une étudiante de l'ENSAD un film d'animation, "Le chercheur et son article» (fig. 3). À partir du témoignage d'un chercheur, ce film montre, avec un graphisme poétique, le processus qui mène de la découverte à la publication.

D'autre part, nous avons développé une collaboration soutenue avec l'école de design ENSCI-Les Ateliers. Nous avons initié trois projets autour de sujets fondamentaux : la supraconductivité, la physique quantique et la lumière. Chaque fois, nous avons suivi pendant quatre mois un groupe de travail formé d'une quinzaine d'étudiants et de deux enseignants-designers. Les étudiants produisent des objets pédagogiques, des vidéos, des affiches, des photos, mais aussi des réalisations plus artistiques ou de design. Ces dispositifs sont ensuite utilisés dans des évènements grand public et sur internet. Ainsi, les vidéos du projet «Design Quantique "sont utilisées dans des conférences en lycée comme porte d'entrée pour amener en douceur les élèves aux explications plus "dures ». Une exposition issue du projet a été présentée à la Cité des Sciences, puis dans des médiathèques et des lycées. Enfin, un site web (www.designquantique.fr) permet au public de découvrir l'intégralité de nos productions.

Derniers exemples, cette fois du côté de la pédagogie : "Option vulgarisation " et "Open TP » sont deux enseignements développés dans le cadre du Magistère de Physique d'Orsay, nouveaux par leurs formats et leurs contenus pédagogiques. Leurs structures s'inspirent directement des modes d'enseignement observés dans des écoles de design ou d'art lors de nos collaborations. Dans l'option vulgarisation, nous proposons aux étudiants de développer

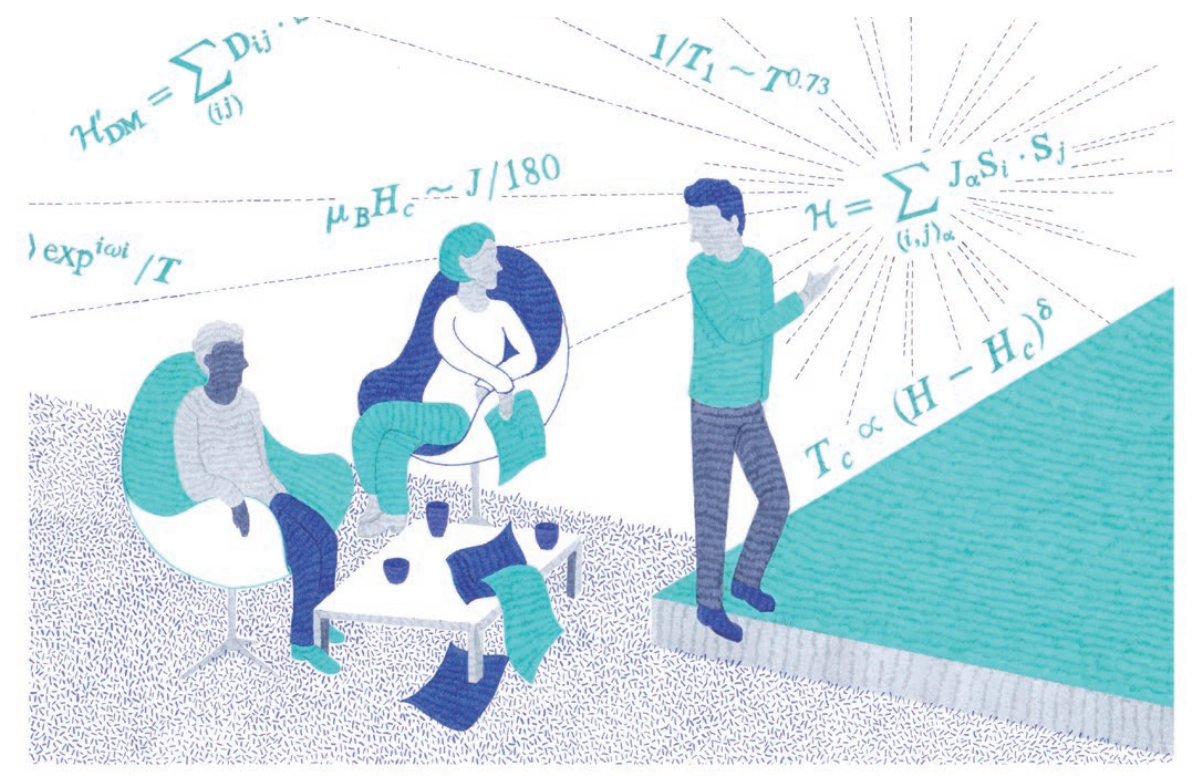

Lors de petites réunions plus ou moins formelles, dans un bureau autour de datas, ça part un peu dans tous les sens et il y a souvent un moment où tu dis «ah oui ! là on tient quelque chose l », ça arrive de façon assez systématique ; il y a dans les sujets, un moment où quelque chose se déclenche, ce n'est pas forcément la fin, ça $n^{\prime}$ induit pas que tu as tout compris, mais tu passes d'une situation très floue où tu es vraiment dans le brouillard à une cristallisation.

2. Une des illustrations de Physiciens des Solides, recueil illustré de témoignages de chercheurs par C. Passavant.

eux-mêmes des projets de vulgarisation. Dans "Open TP ", nous proposons des séances de travaux pratiques de physique, où les étudiants décident de leur propre sujet, puis développent des dispositifs de mesure à l'aide de Legos, de cartes et de capteurs à bas coût "Arduino ", ainsi que d'autres outils de base. Par exemple, les étudiants ont mesuré la résistance de diodes électroluminescentes en fonction de la température, ou un cycle d'aimantation de supraconducteurs. Nous avons également développé un ensemble d'outils graphiques qui permettent aux étudiants, à la fin des séances, de fabriquer une petite bande dessinée en guise de mode d'emploi de leur TP, qu'ils peuvent partager en ligne. Nous espérons ainsi développer un échange avec des étudiants d'autres universités qui suivraient la même démarche. En effet, l'idée d'une communauté de partage, un peu dans l'esprit Wikipédia, semble séduire et motiver ces nouvelles générations d'étudiants, et nous permet de renouveler profondément notre approche des travaux pratiques de physique.

\section{En quoi ces productions sont-elles originales?}

Souvent, les outils de vulgarisation développés par notre communauté conviennent à des actions locales, comme la Fête de la Science. Mais la bonne volonté ne suffit pas pour réaliser des outils visant une large diffusion. Et c'est bien normal, car un physicien n'est ni graphiste, ni designer. Notre groupe choisit plutôt de faire appel à des professionnels quand c'est nécessaire : graphistes, designers, illustrateurs... Nous ne " commandons " pas seulement des produits. Nous développons plutôt un processus de coconstruction avec des allers-retours constants, voire parfois en organisant la présence sur une longue durée des illustrateurs ou des designers dans notre laboratoire. Car c'est justement en travaillant ensemble, physiciens et spécialistes de la forme, à l'interface entre disciplines scientifiques et artistiques, que nous réussissons à allier la forme et le fond, et à produire des objets nouveaux.

Quel est l'accueil du public ? Pour le savoir, nous nous investissons dans de nombreuses actions grand public, aidés par des doctorants en missions doctorales. Nous multiplions ainsi les occasions de tester ces nouveaux modes de vulgarisation autour de la matière condensée et de la physique quantique : interventions en lycées et en classes préparatoires, expositions dans des musées de science et d'autres lieux plus originaux comme les restaurants universitaires, conférences grand public, organisations d'évènements comme la journée quantique au Musée des Arts \& Métiers avec le CNRS... Les retours par 


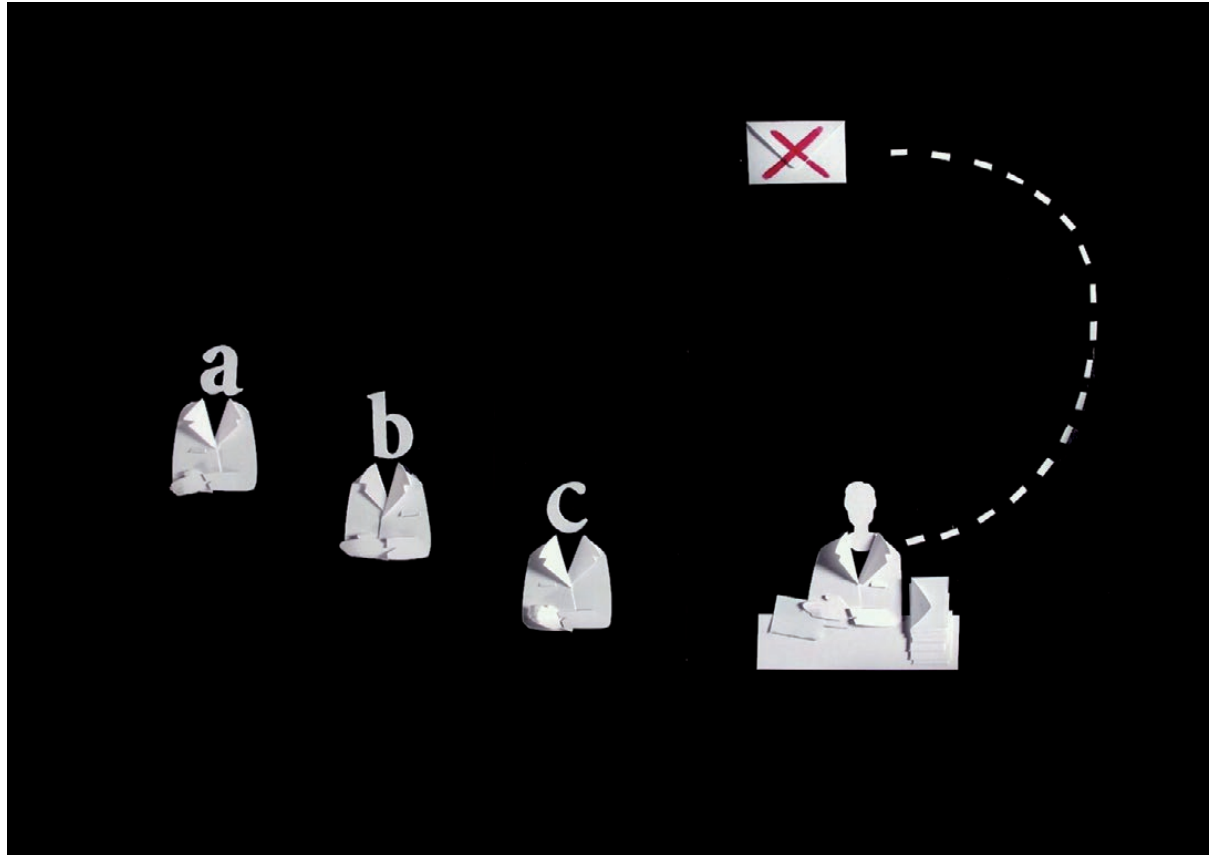

3. " Le chercheur et son article : une aventure en trois actes ", un film d’animation de C. Arene décrivant le processus de recherche menant à une publication. À voir sur https://vimeo.com/108367620

sondage et les témoignages sont très positifs. Mais une évaluation plus rigoureuse et scientifique reste nécessaire.

Ces productions et actions grand public jouent souvent sur des registres ludiques, esthétiques, et visent à créer un autre rapport à la physique. Elles servent de premier pas vers des explications plus rigoureuses. Par exemple, dans un lycée, une vidéo humoristique sur l'effet tunnel constitue une bonne introduction pour un exposé scientifique sur la physique quantique. Le Physics Circus (fig. 1) permet d'engager un dialogue avec le public sur l'origine de la lévitation supraconductrice ; les mouvements des animaux supraconducteurs permettent alors de discuter de l'ancrage par les vortex. Ces formats complètent d'autres approches plus approfondies, par exemple les actions en milieu scolaire comme "La main à la pâte ", "Les Savanturiers " ou "Un chercheur, un enseignant, une classe ", ou les livres de vulgarisation.

Pour aller plus loin, nous développons depuis peu des liens avec des chercheurs en sciences humaines et sociales pour mieux comprendre et évaluer à la fois l'efficacité de nos contenus et ce qui se joue dans leur mode de construction. Quel type de vulgarisation produisent des physiciens quand ils travaillent avec des designers ? C'est une des questions de recherche auxquelles nous allons tenter de répondre dans le cadre de l'ANR DeSciTech (Sciences, design et société : la fabrique des mondes contemporains) qui vient de débuter, où collaboreront des physiciens comme Joël Chevrier (Université Joseph Fourier), des designers de l'ENSCI, et des chercheurs en sciences humaines et sociales (Télécom-ParisTech, Université Paris-Est).

Le public ne connait souvent la physique moderne qu'à travers quelques physiciens médiatiques qui l'incarnent à l'écran ou dans des livres, comme Hubert Reeves : ces figures charismatiques, certes utiles, servent parfois d'excuse aux autres physiciens pour ne pas s'impliquer. Notre démarche vise plutôt à reproduire ce qui paraît si naturel en recherche fondamentale : la construction collective de pratiques et de savoirs pour que rien ne se perde et que toute invention ou découverte puisse servir à tous, par la publication et le partage. Voilà pourquoi nous associons nos collègues à nos projets et aux évènements publics ; nous proposons aussi des formations aux autres laboratoires et aux musées de science. Surtout, nous tentons de développer un réseau d'échanges de bonnes pratiques de vulgarisation et de mutualisation des moyens. Car plus nous serons nombreux, plus nous gagnerons en efficacité et en reconnaissance.

\section{Plus nombreux, plus forts}

Mais, malgré la bienveillance de nos institutions pour ces actions grand public, les modes de recrutement et de promotions freinent l'implication des chercheurs. Vaut-il mieux une Physical Review Letters de plus à un dossier de candidature qu'un engagement en vulgarisation ? Malgré le soutien croissant des directions d'instituts ou de laboratoires pour ces actions, il nous semble que les chercheurs hésitent parfois à s'impliquer. Un travail de pédagogie s'impose vis-à-vis de nos collègues et des commissions d'évaluation pour expliquer le sens de ces actions de vulgarisation, leur intérêt pour nos laboratoires et notre communauté.

Nous devons associer encore plus de chercheurs à ces actions. On constate en effet que les chercheurs qui ont déjà collaboré avec nous portent un regard différent sur ce type d'actions. Autre piste : mieux noter, dans les demandes de financements de recherche type ANR, les chercheurs qui proposent un vrai volet " grand public". C'est déjà le cas, mais sans grande efficacité, car l'évaluation de ces actions n'a pas la même rigueur que pour le volet « recherche». Améliorer le sérieux de cette évaluation, et ainsi la prise en compte des actions de vulgarisation dans les demandes de financement, irait dans le bon sens.

Grâce à ces actions, nous espérons d'une part convaincre plus de chercheurs à participer à des actions de vulgarisation, et d'autre part aider à la production de dispositifs plus professionnels. Pour cela, une réelle volonté de notre propre communauté, mais aussi des instances politiques, est plus que jamais nécessaire pour faire changer les choses en profondeur.

Nous espérons qu'avec cet article, d'autres physiciens découvriront et adhéreront à cette démarche, et qu'ensemble nous aurons un réel impact. L'enjeu est crucial : il s'agit de construire ensemble l'image que nous souhaitons donner de la physique moderne et de la recherche!

\section{Références}

Toutes nos activités et productions sont présentées sur le site www.vulgarisation.fr

Cécile Michaut, Vulgarisation scientifique, mode d'emploi, EDP Sciences (2014). 